$19^{\text {th }}$ European Symposium on Computer Aided Process Engineering - ESCAPE19

J. Jeżowski and J. Thullie (Editors)

(c) 2009 Elsevier B.V./Ltd. All rights reserved.

\title{
Particle Swarm Optimization for Phase Stability and Equilibrium Calculations in Reactive Systems
}

\author{
Adrián Bonilla-Petriciolet ${ }^{\mathrm{a}}$ and Juan Gabriel Segovia-Hernández ${ }^{\mathrm{b}}$ \\ ${ }^{a}$ Departamento de Ingeniería Química, Instituto Tecnológico de Aguascalientes, Av. \\ López Mateós 1801, Aguascalientes, 20256, México.petriciolet@hotmail.com \\ ${ }^{b}$ Departamento de Ingeniería Química, División de Ciencias Naturales y Exactas, \\ Universidad de Guanajuato, Campus Guanajuato, Noria Alta s/n, Guanajuato, 36050, \\ México.gsegovia@quijote.ugto.mx
}

\begin{abstract}
This study reports the application of Particle Swarm Optimization (PSO) for phase stability and equilibrium calculations in reactive systems. These thermodynamic problems are formulated using transformed composition variables and PSO is used as global optimization strategy. Our results indicate that Particle Swarm Optimization is an alternative and suitable stochastic method for solving these challenging calculations in reactive systems.
\end{abstract}

Keywords: Particle swarm optimization, phase equilibrium, chemical equilibrium, global optimization

\section{Introduction}

In recent years, reactive separation processes have emerged as attractive technologies for the chemical industry because they offer various advantages over conventional separation strategies. The design and synthesis of these separation units is fundamentally based on the study and analysis of thermodynamic equilibrium. However, the modeling of phase equilibrium in reactive systems is a difficult task due to the complex interactions among components, phases and reactions. In these conditions, conventional numerical methods are not suitable for performing reactive phase equilibrium calculations. Until now, some studies have applied global solving strategies for reliably modeling the phase behavior in reactive systems; see for example McDonald and Floudas (1996) and Bonilla-Petriciolet et al. (2006). Stochastic methods have proven to be useful because they do not require assumptions about the thermodynamic problem, are reliable and efficient. However, few attempts have been performed on the application of these strategies for reactive phase equilibrium calculations. One of the most-promising stochastic methods is Particle Swarm Optimization (PSO) (Kennedy and Eberhart, 1995). It is a novel evolutionary algorithm capable of handling the challenging characteristics of global optimization problems. Furthermore, PSO is simpler, both in formulation and computer implementation, than the Genetic Algorithm and other metaheuristics. In the chemical engineering community, PSO is of great interest and has been successfully used in several applications. However, the performance of PSO in the modeling of phase behavior of reactive systems has yet to be studied. Therefore, in this study we illustrate the application of PSO for performing phase stability and equilibrium calculations in mixtures subject to chemical reactions. 


\section{Formulation of reactive phase stability and equilibrium problems}

At constant temperature and pressure, a multicomponent and multiphase system achieves equilibrium when its total Gibbs free energy is at the global minimum. For reactive mixtures, the Gibbs free energy minimization is subject to chemical equilibrium restrictions. However, this difficult thermodynamic problem can be readily solved if the Gibbs energy function is expressed in terms of transformed composition variables (Ung and Doherty, 1995). Transformed composition variables provide a simpler thermodynamic framework for modeling reactive mixtures, restrict the solution space to the compositions that satisfy stoichiometry requirements, and reduce the problem dimensionality (Ung and Doherty, 1995; Wasylkiewicz and Ung, 2000). Thus, for a system of $c$ components that undergoes $r$ independent chemical reactions, the transformed mole fractions $X_{i}$ are defined by selecting $r$ reference components

$$
X_{i}=\frac{x_{i}-v_{i} N^{-1} x_{r e f}}{1-v_{T O T} N^{-1} x_{r e f}} \text { for } i=1, \ldots, c-r
$$

where $x_{i}$ is the mole fraction of component $i, x_{\text {ref }}$ is a column vector of mole fractions for $r$ reference components, $v_{i}$ is the row vector of stoichiometric coefficients of component $i$ for each of the $r$ reactions, $N$ is an invertible and square matrix formed from the stoichiometric coefficients of the reference components in the $r$ reactions, and $v_{T O T}$ is a row vector where each element corresponds to the sum of stoichiometric coefficients for all components that participate in each of the $r$ reactions. Note that transformed mole fractions $(X)$ are related to conventional mole fractions $(x)$ using the reaction equilibrium constants (Ung and Doherty, 1995). Using these transformed variables, the Gibbs free energy function for a multiphase system is given by

$$
\hat{g}=\sum_{j=1}^{\pi} \Phi_{j} \sum_{i=1}^{c-r} X_{i, j} \mu_{i, j}
$$

where $\hat{g}$ is the transformed molar Gibbs free energy, $\pi$ is the number of phases at equilibrium, $\mu_{i, j}$ is the chemical potential of component $i$ in phase $j, X_{i, j}$ is the transformed mole fraction of component $i$ in phase $j$, and $\Phi_{i, j}$ is the transformed mole fraction for phase $j$. Transformed composition variables are subject to the material balances

$$
\begin{aligned}
& Z_{i}-\sum_{j=1}^{\pi} X_{i, j}=0 \text { for } i=1, \ldots, c-r \\
& \sum_{i=1}^{c-r} X_{i, j}=1 \text { for } j=1, \ldots, \pi \\
& \sum_{j=1}^{\pi} \Phi_{j}=1
\end{aligned}
$$

where $Z_{i}$ is the transformed mole fraction of component $i$ in the feed. For a reactive mixture with all $X_{i} \in(0,1)$, the unconstrained global optimization of Eq. (2) can be performed using the next equations 
$\hat{n}_{i, j}=\lambda_{i, j}\left(Z_{i}-\sum_{m=1}^{\pi-1} \hat{n}_{i, m}\right)$ for $i=1, \ldots, c-r$ and $j=1, \ldots, \pi-1$

$\hat{n}_{i, \pi}=Z_{i}-\sum_{m=1}^{\pi-1} \hat{n}_{i, m}$ for $i=1, \ldots, c-r$

where $\lambda_{i, j} \in(0,1)$ for $i=1, \ldots, c-r$ and $j=1, \ldots, \pi-1$ are the decision variables and $\hat{n}_{i, j}$ is the transformed mole number of component $i$ in phase $j$. Note that

$X_{i, j}=\frac{\hat{n}_{i, j}}{\sum_{k=1}^{c-r} \hat{n}_{k, j}}$

$\Phi_{j}=\frac{\sum_{k=1}^{c-r} \hat{n}_{k, j}}{\sum_{m=1}^{\pi} \sum_{k=1}^{c-r} \hat{n}_{k, m}}$ for $i=1, \ldots, c-r$ and $j=1, \ldots, \pi$

On the other hand, for a reactive mixture, the phase stability analysis can be performed via the global minimization of the reactive tangent plane distance function (RTPDF). This stability criterion is also formulated using $X$ and, as a consequence, retains all characteristics and advantages of the classical stability test used for non-reactive mixtures. So, RTPDF is defined as (Wasylkiewicz and Ung, 2000)

$R T P D F=\sum_{i=1}^{c-r} X_{i}\left(\left.\mu_{i}\right|_{X}-\left.\mu_{i}\right|_{Z}\right)$

where $\mu_{i}$ is the chemical potential of component $i$ evaluated at the transformed composition $X$ or $Z$. The necessary and sufficient condition for global stability is $R T P D F \geq 0$ for any transformed composition $X$ from the whole composition space. $R T P D F$ is optimized with respect to $c-r$ decision variables $\lambda_{i, 1} \in(0,1)$ using Eq. (4) where $X$ is obtained from Eq. (5). Finally, it is convenient to note that the chemical potentials are functions of $X$ for both $\hat{g}$ and $R T P D F$.

\section{Optimization strategy: Particle Swarm Optimization}

Particle Swarm Optimization is a population based method that belongs to the class of swarm intelligence algorithms. Eberhart and Kennedy (1995) introduced this strategy for global optimization, which is inspired by the social behavior of flocking swarms of birds and fish schools. It exploits a population of potential solutions to identify promising areas for optimization. In this context, the population of potential solutions is called the swarm, and each solution is called particle. The success histories of the particles influence both their own search patterns and those of their peers. Each particle has two state variables: its current position $s_{i, j}(t)$ and its current velocity $V_{i, j}(t)$ where $t$ is an iteration counter. In the local version of PSO, the search is focused on promising regions by biasing each particle's velocity toward both the particle's own remembered 
best position $\left(s_{i, j}^{p}\right)$ and the communicated best neighborhood location $\left(s_{i, j}^{\text {best }}\right)$. The relative weights of these two positions are scaled by the social $\left(C_{1}\right)$ and cognitive $\left(C_{2}\right)$ parameters. The velocity and position of each particle are updated by using:

$V_{i, j}(t+1)=w V_{i, j}(t)+C_{1} R_{1}\left(s_{i, j}^{\text {best }}-s_{i, j}(t)\right)+C_{2} R_{2}\left(s_{i, j}^{p}-s_{i, j}(t)\right)$

$s_{i, j}(t+1)=s_{i, j}(t)+V_{i, j}(t+1)$

where $R_{1}, R_{2} \in(0,1)$ are random numbers, and $w$ is the inertia weight factor. In our calculations, we have considered that $w$ decreases linearly from 0.8 to 0.4 over the whole run; while $C_{1}$ also decreases linearly from 2.0 to 0.5 where $C_{2}=4.0-C_{1}$. Dynamic values for $w, C_{1}$ and $C_{2}$ are used to favor the global search during the early iterations and to encourage the particles to converge to global optimum at the end of the search. The velocity of each particle is restricted to a maximum value within the interval $\left[-V_{\max }, V_{\max }\right]$, which is defined by considering the bounds on decision variables. Each particle is assigned to a neighborhood of a pre-specified number of particles $\left(n_{h}=10\right)$. Thus, the best position attained by particles that comprise the neighborhood is communicated among them. It is important to note that all PSO parameters were defined by considering the results obtained from several trial calculations using benchmark problems and our numerical experience. Finally, the overall algorithm of PSO is given as follows: at the beginning, a population of $n_{p}$ particles is initialized with random positions $s_{i, j}$ and random velocities $V_{i, j}$ for $i=1, \ldots, n_{v a r}$ and $j=1, \ldots, n_{p}$ where $n_{v a r}$ is the number of decision variables. Once all particles are initialized, the positions and velocities of all particles are modified using Eq. (7). After calculating the velocities and position for the next iteration $t+1$, the current iteration is completed. The best particle is only updated when a new one is found yielding a decrease in the objective function value. This process is performed for a certain number of iterations $\left(N_{\text {iter }}\right)$, or until a maximum number of iterations, without improvement in the best function value, is satisfied.

\section{Results and Discussion}

The reaction for butyl acetate production at $298.15 \mathrm{~K}$ and $1 \mathrm{~atm}$ is used to illustrate the performance of PSO. This reactive system is given by: acetic acid (1) + n-butanol (2) $\leftrightarrow$ water (3) + n-butyl acetate (4). Phase stability and equilibrium calculations are performed for a transformed feed $Z(0.05,0.2,0.75)$. At these conditions, this mixture shows a liquid-liquid equilibrium, and UNIQUAC model is used to predict thermodynamic properties with the parameters reported by Wasylkiewicz and Ung (2000). Transformed fractions $X$ are defined using n-butyl acetate as reference component

$$
\begin{aligned}
& X_{1}=x_{1}+x_{4} \\
& X_{2}=x_{2}+x_{4} \\
& X_{3}=x_{3}-x_{4}=1-X_{1}-X_{2}
\end{aligned}
$$

Both RTPDF and $\hat{g}$ are minimized by considering three decision variables: $\lambda_{i, 1} \in(0,1)$. Phase stability and equilibrium problems were solved 100 times using random initial values via different random number seed. For illustrative purposes, Figure 1 shows the plot of success ratio (SR) versus $N_{\text {iter }}$ for PSO in this system. Note that SR is defined as the number of runs out of 100 that satisfy the condition $\left|f_{\text {opt }}-f_{\text {calc }}\right| \leq 10^{-04}$ where the 
known global optimum of the objective function is $f_{\text {opt }}$, and $f_{\text {calc }}$ is the value of objective function calculated by PSO. This plot was obtained using the best objective function values recorded at different values of $N_{i t e r}$ and for several swarm sizes $n_{p}$.
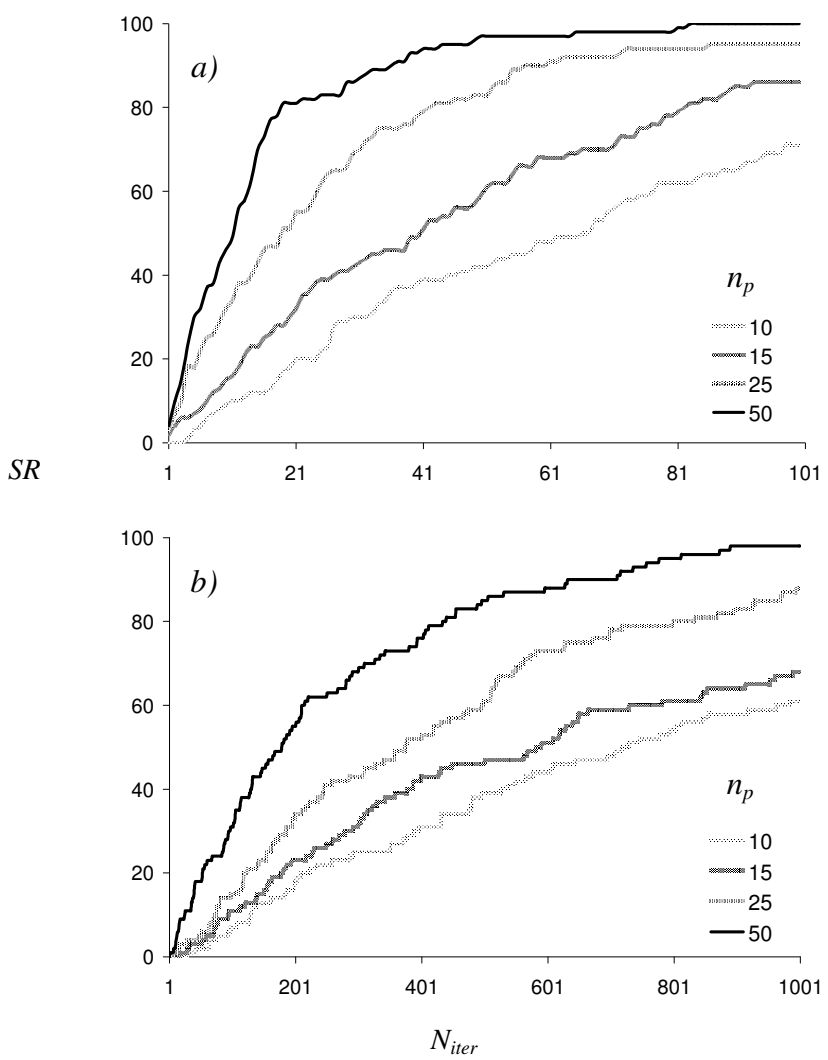

Figure 1. Success ratio versus number of iterations of PSO for the global optimization of: (a) $R T P D F$ and (b) $\hat{g}$. Reactive mixture: reaction for butyl acetate production at $298.15 \mathrm{~K}$ and $1 \mathrm{~atm}$.

Results indicate that the algorithm performance (reliability and efficiency) is dependent on $N_{i t e r}$ and $n_{p}$. Reliability of PSO improves as these parameters increase but at the expense of a higher computational effort. In stability calculations for this example, high SR is obtained at $N_{i t e r}>100$ and $n_{p}>25 n_{\text {var }}$. However, for $\hat{g}$, several iterations are required to obtain an acceptable performance and the increase of $n_{p}$ favors the reliability of PSO. As expected, reactive phase equilibrium problems are generally more difficult to solve compared to stability problems, and they involve more numerical effort. It is expected that for reactive phase equilibrium problems with more decision variables (i.e., multireactive and multicomponent mixtures), the reliability of PSO will increase with $N_{\text {iter }}$ and $n_{p}$. For this example, CPU time ranged from 0.6 to $60 \mathrm{~s}$, depending on the swarm size and maximum number of iterations. Based on our numerical practice using PSO, it appears that this stochastic method is a reliable strategy for modeling the phase 
behavior in reactive systems. The comparison of PSO with other stochastic methods is under way by our research group.

\section{Conclusions}

This paper introduces the application of Particle Swarm Optimization for performing phase stability and equilibrium calculations in reactive systems. This stochastic method appears to be robust for solving these challenging thermodynamic problems in a reasonable computational time. In future studies, we will hybridize this method with other metaheuristics to develop more efficient global optimization strategies for these calculations.

\section{Acknowledgements}

We acknowledge the financial support provided by CONACyT, Dirección General de Educación Superior Tecnológica, Instituto Tecnológico de Aguascalientes and Universidad de Guanajuato (Mexico).

\section{References}

C.M. McDonald, C.A. Floudas, 1996, GLOPEQ: A new computational tool for the phase and chemical equilibrium problem, Computers Chemical Engineering, 21, 1, 1-23.

A. Bonilla-Petriciolet, R. Vazquez-Roman, G.A. Iglesias-Silva, K.R. Hall, 2006, Performance of stochastic global optimization methods in the calculation of phase stability analyses for nonreactive and reactive mixtures, Industrial Engineering Chemistry Research, 45, 13, 47644772 .

J. Kennedy, R.C. Eberhart, 1995, Particle Swarm Optimization, In Proceedings of the IEEE International Conference on Neural Networks, IV: 1942-1948.

S. Ung, M.F. Doherty, 1995, Theory of phase equilibrium in multireaction systems, Chemical Engineering Science, 50, 20, 3201-3216.

S.K. Wasylkiewicz, S. Ung, 2000, Global phase stability analysis for heterogeneous reactive mixtures and calculation of reactive liquid-liquid and vapor-liquid-liquid equilibria, Fluid Phase Equilibria, 175, 1-2, 253-272. 